\title{
PREVALENCE OF GENERIC LAVER DIAMOND
}

\author{
SEAN D. COX
}

\begin{abstract}
Viale [19] introduced the notion of Generic Laver Diamond at $\kappa$ which we denote $\nabla_{\text {Lav }}(\kappa)$-asserting the existence of a single function from $\kappa \rightarrow$ $H_{\kappa}$ that behaves much like a supercompact Laver function, except with generic elementary embeddings rather than internal embeddings. Viale proved that the Proper Forcing Axiom (PFA) implies $\diamond_{\text {Lav }}\left(\omega_{2}\right)$. We strengthen his theorem by weakening the hypothesis to a statement strictly weaker than PFA. We also show that the principle $\diamond_{\text {Lav }}(\kappa)$ provides a uniform, simple construction of 2-cardinal diamonds, and prove that $\nabla_{\text {Lav }}(\kappa)$ is quite prevalent in models of set theory; in particular:

(1) $L$ satisfies $\diamond_{\text {Lav }}^{+}(\kappa)$ whenever $\kappa$ is a successor cardinal, or when the appropriate version of Chang's Conjecture fails.

(2) For any successor cardinal $\kappa$, there is a $\kappa$-directed closed class forcingnamely, the forcing from Friedman-Holy [10] - that forces $\diamond_{\text {Lav }}(\kappa)$.
\end{abstract}

Prediction principles have been central topics in set theory ever since Jensen introduced the $\diamond$ principle in the 1960s. Not only does $\diamond$ hold in canonical inner models such as $L$, it also is frequently introduced by forcing; for example, adding a Cohen subset of $\kappa$ introduces a $\nabla_{\kappa}$ sequence. The $\diamond$ principle is frequently used to prove one direction of an independence result 1 Two-cardinal variations of $\diamond$-i.e. versions of $\diamond$ which guess subsets of some fixed $\wp_{\kappa}(\lambda)$, rather than just guessing subsets of $\kappa$-were introduced by Jech [8]. Donder-Matet [5] (with a correction by Shioya [17]) proved that mild cardinal arithmetic assumptions are enough to guarantee such 2-cardinal versions of $\diamond$; for example, $\kappa^{<\kappa}=\kappa$ implies that $\diamond(\kappa, \lambda)$ holds for all $\lambda \geq \kappa^{+}$.

Laver [13] proved that if $\kappa$ is a supercompact cardinal, then there is a function $F: \kappa \rightarrow H_{\kappa}$ which essentially behaves like a universal $\nabla_{\kappa}$ sequence with respect to supercompactness measures (rather than merely with respect to the club filter, as is the case with usual $\nabla_{\kappa}$ and its 2-cardinal variants). For this reason it is commonly called a Supercompact Laver Diamond/Function, and notably appears in the consistency proofs of the Proper Forcing Axiom, Martin's Maximum, and an indestructibly supercompact cardinal.

The concept of a Laver function has been generalized in two distinct directions; unfortunately both are referred to as "Generalized Laver Diamond" in the literature:

2010 Mathematics Subject Classification. 03E57 03E55 03E35 03E05 .

In memory of Richard Laver, 1942-2012.

Part of this work was done while the author participated in the Thematic Program on Forcing and its Applications at the Fields Institute, which was partially supported from NSF grant DMS1162052.

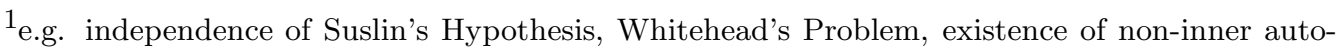
morphisms of the Calkin algebra, Naimark's Problem, and many others. 
(1) Generalizations of (supercompact) Laver Diamond to some other large cardinal notions (e.g. strong cardinals) have been developed (e.g. [11, [2], 6], and [7]).

(2) Viale [19] generalized the notion of Laver Diamond so that it makes sense at successor cardinals.

To distinguish Viale's version from the large cardinal versions mentioned above, we will refer to Viale's version as "Generic" Laver Diamond at $\kappa$, denoted by $\diamond_{\text {Lav }}(\kappa)$. This is a function from $\kappa \rightarrow H_{\kappa}$ which behaves somewhat like a Laver function, except with generic rather than internal ultrapowers (it can also be defined without reference to forcing; see Section 10. In particular, $\diamond_{\text {Lav }}(\kappa)$ can hold when $\kappa$ is a successor cardinal, and $\diamond_{\text {Lav }}(\kappa)$ provides a particularly elegant, uniform way to produce two-cardinal diamond sequences; see Section 3 for such a construction. Viale's main result was that the Proper Forcing Axiom (PFA) implies $\diamond_{\text {Lav }}\left(\omega_{2}\right)$. We strengthen this theorem by weakening the hypothesis to a statement strictly weaker than PFA; moreover our proof is completely elementary and does not make use of the coding of $H_{\omega_{2}}$ which appeared in Viale's proof 2

Theorem 1. If $\mathfrak{c}=\omega_{2}$ and the class $G I C_{\omega_{1}}$ of $\omega_{1}$-guessing, internally club sets is stationary, then $\nabla_{\text {Lav }}\left(\omega_{2}\right)$ holds.

More generally: whenever $\Gamma \subset \wp_{\kappa}(V)$ is a $\Pi_{1}(V)$ definable stationary class in some parameter from $H_{\kappa^{+}}$such that:

- $\Gamma$ projects downward; and

- $\Gamma$ satisfies the Isomorphism Property

then there is a $\diamond_{\text {Lav }}(\Gamma)$ function.

Our proof of Theorem 1 gives an explicit construction of the $\diamond_{\text {Lav }}(\Gamma)$ function; namely, the function defined recursively in Claims 33.1 and 33.2 .

We also prove - as is the case with the weaker $\nabla_{\kappa}$ and its 2-cardinal variants - that the principle $\diamond_{\text {Lav }}(\kappa)$ is quite prevalent in models of set theory:

Theorem 2. If Stationary Condensation holds at $\kappa$ and $\kappa$ is a successor cardinal, then $\diamond_{\text {Lav }}(\kappa)$ holds. More generally, if the appropriate version of weak Chang's Conjecture fails at $\kappa$, then $\diamond_{\text {Lav }}(\kappa)$ holds.

Combining this theorem with the results of Friedman-Holy [10] yields:

Corollary 3. If $\kappa$ is a successor cardinal, then there is a $\kappa$-directed closed class forcing extension that models $\diamond_{\text {Lav }}(\kappa)$.

We also prove:

Theorem 4. L satisfies $\diamond_{\text {Lav }}^{+}(\kappa)$ whenever $\kappa$ is a successor cardinal, or whenever the appropriate version of weak Chang's Conjecture fails at $\kappa$. By Theorem $14 \nabla_{\text {Lav }}(\kappa)$ also holds for such $\kappa$.

\footnotetext{
${ }^{2}$ The hypothesis that $\mathfrak{c}=\omega_{2}$ and GIC $_{\omega_{1}}$ is a stationary class is a consequence of PFA (by VialeWeiß [20] and Todorcevic [18]), but is strictly weaker than PFA; see Remark 33 In particular it does not imply the existence of the Caicedo-Velickovic coding.
} 
The paper is structured as follows: Section 1 provides the definitions and basic facts about $\nabla_{\text {Lav }}(\kappa)$ and its variants; Section 2 proves that $\nabla_{\text {Lav }}(\kappa)$ is equivalent to $\diamond_{\text {Lav }}^{-}(\kappa)$; Section 4 proves Theorems 2 and 4. Section 5 proves Theorem 1; and Section 6 concludes with a question.

We will use the following notation throughout the paper:

Definition 5. Suppose $X$ is a set and $(X, \in \uparrow(X \times X))$ is extensional $3^{3}$ Then $H_{X}$ denotes the transitive collapse of $X$ and $\sigma_{X}: H_{X} \rightarrow X$ denotes the inverse of the Mostowski collapsing map of $X$.

We also will use the following convention:

Convention 6. If $p_{1}, \ldots, p_{n}$ are each elements of some $H_{\theta}$, then each $p_{i}$ in the structure $\left(H_{\theta}, \in, p_{1}, \ldots, p_{n}\right)$ will be understood to be the natural interpretation of a constant symbol $\dot{p}_{i}$.

The author would like to thank the anonymous referee for many helpful suggestions, especially regarding the structure of the proof of Theorem 14 .

$$
\text { 1. } \diamond_{\text {LAV }}(\Gamma), \diamond_{\text {LAV }}^{+}(\Gamma), \text { AND } \diamond_{\text {LAV }}^{-}(\Gamma)
$$

We recall the definition of Generic Laver Diamond from Viale [19]: a function $F: \kappa \rightarrow H_{\kappa}$ is a Generic Laver function iff for every set $b$ and for every sufficiently large regular $\theta$, there are stationarily many $M \in \wp_{\kappa}\left(H_{\theta}\right)$ such that $b \in M$ and the Mostowski collapse of $M$ sends $b$ to $F(M \cap \kappa)$ The role of the class $\Gamma$ in the following definition is to allow for refinements; similarly to the way that $\diamond(S)$ refines $\nabla_{\kappa}$ (where $S$ is a stationary subset of $\kappa$ ).

Definition 7. Suppose $\kappa$ is a regular uncountable cardinal and $F: \kappa \rightarrow V$ is a function. If $b$ is a set and $\theta$ is a regular uncountable cardinal, define $G_{=F}^{b, \theta}$ to be the set of those $M$ such that:

(a) $b \in M \prec\left(H_{\theta}, \in\right)$

(b) $M \cap \kappa \in \kappa$

(c) $\sigma_{M}^{-1}(b)=F(M \cap \kappa)$ (recall that $\sigma_{M}: H_{M} \rightarrow M$ denotes the inverse of the Mostowski collapse of $M$ )

For any class $\Gamma$, we say that $F$ is a $\diamond_{\text {Lav }}(\Gamma)$ function iff for every set $b$ and every sufficiently large regular $\theta$, the following set is stationary:

$$
\Gamma \cap G_{=F}^{b, \theta}
$$

We say that $\diamond_{\text {Lav }}(\Gamma)$ holds iff there is a $\diamond_{\text {Lav }}(\Gamma)$ function.

We also define variants of Generic Laver Diamond which are analogous to what Kunen [12] calls $\diamond^{-}$and $\diamond^{+}$:

Definition 8. Suppose $\kappa$ is a regular uncountable cardinal and $F: \kappa \rightarrow V$ is a function. For each set $b$ and regular uncountable $\theta$, the set $G_{\in F}^{b, \theta}$ is defined the same

\footnotetext{
${ }^{3}$ e.g. if $X \prec\left(H_{\theta}, \in\right)$ for some $\theta$.

${ }^{4}$ Viale's formulation was actually more similar to the characterization in Lemma 12 below.
} 
way that $G_{=F}^{b, \theta}$ was defined in Definition 7 except requirement (c) is replaced with the following requirement:

$$
\text { (c), } \sigma_{M}^{-1}(b) \in F(M \cap \kappa)
$$

For any class $\Gamma$, we say that $F$ is a $\diamond_{\text {Lav }}^{-}(\Gamma)$ (resp. $\diamond_{\text {Lav }}^{+}(\Gamma)$ ) function iff

(A) For every set $b$ and all sufficiently large regular $\theta: \Gamma \cap G_{\in F}^{b, \theta}$ is stationary (resp. contains all but nonstationarily many elements of $\Gamma \cap \wp\left(H_{\theta}\right)$ )

(B) $|F(\alpha)| \leq|\alpha|$ for every $\alpha<\kappa$.

We say $\diamond_{\text {Lav }}^{-}(\Gamma)$ (resp. $\diamond_{\text {Lav }}^{+}(\Gamma)$ ) holds iff there exists $a \diamond_{\text {Lav }}^{-}(\Gamma)$ (resp. $\diamond_{\text {Lav }}^{+}(\Gamma)$ ) function.

Remark 9. In the special case where $\Gamma$ is the natural class $\wp_{\kappa}(V)$, we will just write $\diamond_{\text {Lav }}(\kappa)$ to mean $\diamond_{\text {Lav }}(\Gamma)$, and similarly for $\diamond_{\text {Lav }}^{-}(\kappa)$ and $\diamond_{\text {Lav }}^{+}(\kappa)$.

There are obvious local variations of Definitions 7 and 8 , but we will not make use of such local variations in this paper 5

Remark 10. If $\Gamma \subseteq \wp_{\kappa}(V)$ and $F$ is a $\diamond_{\text {Lav }}(\Gamma)$ function, then so is

$$
F \uparrow\left\{\alpha<\kappa \mid F(\alpha) \in H_{\kappa}\right\}
$$

Proof. Let $b$ be any set. Let $\theta$ be a sufficiently large regular cardinal so that $\Gamma \cap G_{=F}^{b, \theta}$ is stationary. Since $\Gamma \subseteq \wp_{\kappa}(V)$ then $H_{M}$ is an element of $H_{\kappa}$ for every $M \in \Gamma \cap G_{=F}^{b, \theta}$; so $F(M \cap \kappa)=\sigma_{M}^{-1}(b) \in H_{M} \in H_{\kappa}$ for any such $M$.

Remark 11. If $\Gamma \subseteq \Gamma^{\prime}$ and $\diamond_{\text {Lav }}(\Gamma)$ holds, then so does $\diamond_{\text {Lav }}\left(\Gamma^{\prime}\right)$. This is analogous to the trivial fact that $\diamond(S)$ implies $\diamond\left(S^{\prime}\right)$ whenever $S \subseteq S^{\prime}$ are stationary subsets of $\kappa$.

Definition 7 can also be rephrased in terms of generic elementary embeddings, a characterization which more closely resembles the definition of supercompact Laver functions. This is the version which appeared in Viale [19] (albeit in the presence of Woodin cardinals). While the following lemma is technically a second-order scheme, it is also possible to obtain a first-order definition involving generic embeddings 6

Lemma 12. A function $F: \kappa \rightarrow V$ is a $\diamond_{\text {Lav }}(\Gamma)$-function if and only if for every $b$ and every sufficiently large regular $\theta$, there is a generic elementary embedding $j: V \rightarrow N$ with critical point $\kappa$ such that:

(1) $H_{\theta}^{V}$ is an element of the (transitivized) wellfounded part of $N$;

(2) $j\left[H_{\theta}^{V}\right] \in N$;

(3) $j(F)(\kappa)=b$; and

(4) $N \models j\left[H_{\theta}^{V}\right] \in j(\Gamma)$

\footnotetext{
${ }^{5}$ Namely, one could weaken $\diamond_{\text {Lav }}(\Gamma)$ by restricting attention to only those $b$ up to some fixed cardinality.

${ }^{6}$ Namely: in the statement of Lemma 12 if one replaces "there is a generic elementary embedding $j: V \rightarrow N$ " with "there is a normal ideal $\mathcal{I}$ such that $\Vdash_{\left(\mathcal{I}^{+}, \subseteq\right)}$ the ultrapower embedding $j:$ $H_{\left(2^{\theta}\right)^{+}}^{V} \rightarrow_{\dot{G}} \operatorname{ult}\left(H_{\left(2^{\theta}\right)^{+}}^{V}, \dot{G}\right)$ has critical point $\kappa$ and has the following properties ..." then the resulting statement is first order, and the proof is similar to the proof given here.
} 
Proof. Assume $F: \kappa \rightarrow H_{\kappa}$ is a $\diamond_{\text {Lav }}(\Gamma)$ function. Fix some $b$ and let $\theta$ be sufficiently large so that $S:=\Gamma \cap G_{=F}^{b, \theta}$ is stationary (see Definition 7 for the meaning of $G_{=F}^{b, \theta}$ ). Let $\mathcal{I}$ be the restriction of the nonstationary ideal to the stationary set $S$. Let $\mathcal{U}$ be $(V, \wp(S) / \mathcal{I})$-generic and $j: V \rightarrow \mathcal{U} N_{\mathcal{U}}$ the generic ultrapower embedding; here $N_{\mathcal{U}}$ may not be wellfounded, but standard applications of Los' Theorem imply that $H_{\theta}^{V}$ is an element of its (transitivized) wellfounded part and moreover:

- $j\left[H_{\theta}^{V}\right]=[\mathrm{id}]_{\mathcal{U}} \in j(\Gamma)$ (since $S \subseteq \Gamma$ )

- $j(F)(\kappa)=b\left(\right.$ since $\left.S \subseteq G_{=F}^{b, \theta}\right)$

Conversely, suppose $F$ is a function from $\kappa \rightarrow H_{\kappa}$ and for every $b$ and sufficiently large $\theta$ there is a generic embedding $j=j_{b, H_{\theta}}: V \rightarrow N$ satisfying the requirements listed in the statement of the lemma. Let $\mathfrak{A}=\left(H_{\theta}, \in,\{b\}, \ldots\right)$ be arbitrary and let $M^{\prime}:=j\left[H_{\theta}^{V}\right]$. Then $\sigma_{M^{\prime}}=j \uparrow H_{\theta}^{V}$ and $N$ models the following facts about $M^{\prime}$ :

- $M^{\prime} \prec j(\mathfrak{A})$

- $j(F)\left(M^{\prime} \cap j(\kappa)\right)=b=\sigma_{M^{\prime}}^{-1}(j(b))$

- $M^{\prime} \in j(\Gamma)$

By elementarity of $j, V$ believes there is an $M$ such that $M \prec \mathcal{A}, F(M \cap \kappa)=\sigma_{M}^{-1}(b)$, and $M \in \Gamma$.

We will use the following definition; many natural classes (e.g. classes of internally approachable structures, etc.) have this property:

Definition 13. A class $\Gamma$ projects downward iff whenever $X \in \Gamma$ and $\theta \leq \sup (X \cap$ $O R D)$ is a regular uncountable cardinal, then $X \cap H_{\theta} \in \Gamma$.

\section{Equivalence of $\diamond_{\text {Lav }}(\Gamma)$ With $\diamond_{\text {LaV }}^{-}(\Gamma)$}

Theorem 14 below - which is a generalization of Kunen's proof that $\diamond^{-}$is equivalent to $\diamond$ (cf. Theorem 7.14 of [12]) - is crucial to the proof of Theorem 2 ,

Theorem 14. Let $\Gamma$ be a downward-projecting class (in the sense of Definition 13) and $\kappa$ a regular uncountable cardinal, and suppose that $M \cap \kappa \in \kappa$ for every $M \in \Gamma$. Then

$$
\diamond_{\text {Lav }}^{-}(\Gamma) \Longleftrightarrow \diamond_{\text {Lav }}(\Gamma)
$$

Proof. The $\Leftarrow$ direction is trivial, since if $F: \kappa \rightarrow H_{\kappa}$ is a $\diamond_{\text {Lav }}(\Gamma)$ function then easily $\alpha \mapsto\{F(\alpha)\}$ is a $\diamond_{\text {Lav }}^{-}(\Gamma)$ function. Now we prove the $\Rightarrow$ direction. Suppose $F: \kappa \rightarrow V$ witnesses $\diamond_{\text {Lav }}^{-}(\Gamma)$. Let $\left\langle z_{\alpha, i} \mid i<\alpha\right\rangle$ be an enumeration of $F(\alpha)$ for each $\alpha<\kappa$. For each $i<\kappa$ define a function $F_{i}: \kappa \rightarrow H_{\kappa}$ as follows: if $z_{\alpha, i}$ is a function with $i$ in its domain, then let $F_{i}(\alpha):=z_{\alpha, i}(i)$; otherwise $F_{i}(\alpha):=\emptyset$. We finish the proof by showing:

Claim 14.1. There is some $i<\kappa$ such that $F_{i}$ is $a \diamond_{\text {Lav }}(\Gamma)$ function.

Proof. Suppose not; so for every $i<\kappa$ there is some $b_{i}$ and some algebra $\mathfrak{A}_{i}=$ $\left(H_{\theta_{i}}, \in, b_{i}\right)$ such that:

$$
\forall M \in \Gamma M \prec \mathfrak{A}_{i} \Longrightarrow \sigma_{M}^{-1}\left(b_{i}\right) \neq F_{i}(M \cap \kappa)
$$

Let $B:=\left\langle b_{i} \mid i<\kappa\right\rangle$ and fix some regular $\Omega$ such that $B$ and $\left\langle\mathfrak{A}_{i}, b_{i} \mid i<\kappa\right\rangle$ are elements of $H_{\Omega}$. Let $T$ denote the set of $M \in \Gamma$ such that $M \prec \mathfrak{B}:=\left(H_{\Omega}, \in, B, \overrightarrow{\mathfrak{A}}, \vec{b}\right)$ 
and $\sigma_{M}^{-1}(B) \in F(M \cap \kappa) ; T$ is stationary because $F$ is a $\diamond_{\text {Lav }}^{-}(\Gamma)$ function. Now $\sigma_{M}^{-1}(B) \in F(M \cap \kappa)=\left\{z_{M \cap \kappa, i} \mid i<M \cap \kappa\right\}$, so there is some $i_{M}<M \cap \kappa$ such that $\sigma_{M}^{-1}(B)=z_{M \cap \kappa, i_{M}}$. Since $i_{M} \in M$ for all $M \in T$ then by Fodor's Lemma there is a stationary $T^{\prime} \subseteq T$ and some fixed $\hat{i}$ such that $i_{M}=\hat{i}$ for all $M \in T^{\prime}$. Fix an $M \in T^{\prime}$. First observe that

$$
\sigma_{M}^{-1}(B)=\sigma_{M}^{-1}\left(\left\langle b_{i} \mid i<\kappa\right\rangle\right)=\left\langle\sigma_{M}^{-1}\left(b_{i}\right) \mid i \in M \cap \kappa\right\rangle
$$

so in particular $\sigma_{M}^{-1}(B)$ is a function with $\hat{i}$ in its domain. Now

$$
\sigma_{M}^{-1}(B)=z_{M \cap \kappa, i_{M}}=z_{M \cap \kappa, \hat{i}}
$$

and so in particular

$$
\sigma_{M}^{-1}(B)(\hat{i})=z_{M \cap \kappa, \hat{i}}(\hat{i})
$$

So (4) and (2) imply:

$$
\sigma_{M}^{-1}\left(b_{\hat{i}}\right)=z_{M \cap \kappa, \hat{i}}(\hat{i})
$$

Now by the definition of $F_{\hat{i}}$ :

$$
F_{\hat{i}}(M \cap \kappa)=z_{M \cap \kappa, \hat{i}}(\hat{i})
$$

Finally (6) and (5) imply:

$$
F_{\hat{i}}(M \cap \kappa)=\sigma_{M}^{-1}\left(b_{\hat{i}}\right)
$$

On the other hand, since $M \prec \mathfrak{B}$ and $\hat{i} \in M$ then $M_{\hat{i}}:=M \cap H_{\theta_{\hat{i}}} \prec \mathfrak{A}_{\hat{i}}$; moreover $M_{\hat{i}} \in \Gamma$ by our assumption that $\Gamma$ projects downward. So (1) implies:

$$
F_{\hat{i}}\left(M_{\hat{i}} \cap \kappa\right) \neq \sigma_{M_{\hat{i}}}^{-1}\left(b_{\hat{i}}\right)
$$

But $M_{\hat{i}} \cap \kappa=M \cap \kappa$ and $\sigma_{M}^{-1}\left(b_{\hat{i}}\right)=\sigma_{M_{\hat{i}}}^{-1}\left(b_{\hat{i}}\right)$, so (8) implies:

$$
F_{\hat{i}}(M \cap \kappa) \neq \sigma_{M}^{-1}\left(b_{\hat{i}}\right)
$$

which contradicts (7) and completes the proof of the claim.

\section{A simple proof of 2-CARdinal Diamond}

Jech [8] introduced a 2-cardinal Diamond principle, which is a guessing principle for subsets of $\wp_{\kappa}(\lambda)$. Precisely, $\diamond(\kappa, \lambda)$ asserts the existence of a function $\left\langle A_{z}\right| z \in$ $\left.\wp_{\kappa}(\lambda)\right\rangle$ such that for every $A \subset \lambda$, the following set is stationary in $\wp_{\kappa}(\lambda)$ :

$$
\left\{z \in \wp_{\kappa}(\lambda) \mid A \cap z=A_{z}\right\}
$$

Viale's principle $\diamond_{\text {Lav }}(\kappa)$ easily implies that $\kappa^{<\kappa}=\kappa$, which in turn-using a theorem of Donder-Matet [4] and a correction by Shioya [17] -implies that $\diamond(\kappa, \lambda)$ holds for all $\lambda>\kappa \sqrt{7}$ However, there is an especially simple, direct proof of the implication

$$
\diamond_{\text {Lav }}(\kappa) \Longrightarrow \forall \lambda>\kappa \diamond(\kappa, \lambda)
$$

\footnotetext{
$\lambda$.

${ }^{7}$ Donder-Matet [4, with the correction by Shioya [17, proved that $\diamond(\kappa, \lambda)$ holds whenever $2^{<\kappa}<$
} 
which does not require going through the theorem of Donder-Matet and Shioya (though of course their proof is much more general, as it assumes only that $2^{<\kappa}<\lambda$ ). Suppose $F: \kappa \rightarrow H_{\kappa}$ witnesses $\diamond_{\text {Lav }}(\kappa)$, and let $\lambda \geq \kappa$ be any cardinal. For each (extensional) $M \in P_{\kappa}(\lambda)$, recall that $\sigma_{M}$ denotes the inverse of the Mostowski collapsing map of $M$, and set

$$
A_{M}:=\sigma_{M}[F(M \cap \kappa)]
$$

Then $\left\langle A_{M} \mid M \in P_{\kappa}(\lambda)\right\rangle$ is a $\nabla_{\kappa, \lambda}$ sequence: let $A \subseteq \lambda$. By Laverness of $F$, there is a stationary set $S^{\prime}$ of $M^{\prime} \prec\left(H_{\left(2^{\lambda}\right)^{+}}, \in,\{A\}\right)$ such that $\sigma_{M^{\prime}}^{-1}(A)=F\left(M^{\prime} \cap \kappa\right)$. Then for any $M^{\prime} \in S^{\prime}$, setting $M:=M^{\prime} \cap \lambda$ we have:

$$
\begin{array}{r}
A \cap M=A \cap\left(M^{\prime} \cap H_{\lambda}\right)=\sigma_{M^{\prime}}\left(F\left(M^{\prime} \cap \kappa\right)\right) \cap\left(M^{\prime} \cap H_{\lambda}\right) \\
=\sigma_{M^{\prime}}\left[F\left(M^{\prime} \cap \kappa\right)\right]=\sigma_{M}[F(M \cap \kappa)]=A_{M}
\end{array}
$$

Thus, setting

$$
S:=\left\{M^{\prime} \cap \lambda \mid M^{\prime} \in S^{\prime}\right\}
$$

we have that $A \cap M=A_{M}$ for every $M \in S$.

\section{4. $\nabla_{\text {Lav }}(\kappa)$, Condensation, and Weak Chang's Conjecture}

We will describe a natural attempt to define a $\diamond_{\text {Lav }}^{-}(\kappa)$ function, which often works in the presence of Condensation and/or the appropriate failure of Chang's Conjecture. For regular uncountable cardinals $\kappa<\theta$, the Chang's Conjecture $(\theta, \kappa) \rightarrow(\kappa,<\kappa)$ means that for every first order structure $\mathfrak{A}$ on $\theta$ (in a countable language) there is an $M \prec \mathfrak{A}$ such that $|M|=\kappa$ and $|M \cap \kappa|<\kappa$; this is equivalent to saying that

$$
\{M \subset \theta|| M \mid=\kappa \text { and }|M \cap \kappa|<\kappa\}
$$

is a (weakly) stationary set. Weak Chang's Conjecture holds at $\kappa, \theta$-abbreviated ${ }_{\mathrm{wCC}}(\kappa, \theta)$ - holds iff for every every first-order structure $\mathfrak{A}=(\theta, \in, \ldots)$ in a countable language, there are stationarily many $\alpha<\kappa$ such that

$$
\sup \{\operatorname{ot}(X \cap \theta) \mid X \prec \mathfrak{A} \text { and } X \cap \kappa=\alpha\} \geq \alpha^{+}
$$

It is easy to see that decreasing the parameter $\theta$ in $\mathrm{wCC}(\kappa, \theta)$ increases the strength; i.e. $\mathrm{wCC}(\kappa, \theta) \Longrightarrow \mathrm{wCC}\left(\kappa, \theta^{\prime}\right)$ whenever $\theta \leq \theta^{\prime}$. Thus the strongest is when $\theta=\kappa^{+}$; the principle wCC $\left(\kappa, \kappa^{+}\right)$is the well-known "weak Chang's Conjecture at $\kappa$ ", denoted ${ }^{\mathrm{wCC}}(\kappa)$ in Definition 1.6 of Donder-Levinski [4].

Remark 15. Under $V=L$, a cardinal $\kappa$ is ineffable iff $w C C(\kappa)$ holds. See Corollary 1.13 of Donder-Levinski [4].

Remark 16. Standard techniques (e.g. as in Foreman-Magidor [9]) enable reformulation of, say, $w C C(\kappa, \theta)$ which only refers to a single structure. For example, fixing a wellorder $\Delta$ of $H_{\left(2^{\theta}\right)^{+}}$, the principle $w C C(\kappa, \theta)$ is equivalent to saying there are stationarily many $\alpha<\kappa$ such that

$$
\sup \left\{\operatorname{otp}(X \cap \theta) \mid X \prec\left(H_{\left(2^{\theta}\right)^{+}}, \in, \Delta\right) \text { and } X \cap \kappa=\alpha\right\} \geq \alpha^{+}
$$


For a regular uncountable $\kappa$ and a class $\Gamma \subset \wp_{\kappa}(V)$, we say that $\Gamma$ is $<O R D$ stationary (resp. $\Gamma$ is $<O R D$ club) if $\Gamma \cap \wp_{\kappa}\left(H_{\theta}\right)$ is stationary (resp. contains a club) for all sufficiently large $\theta$. Note that $\diamond_{\text {Lav }}(\Gamma)$ trivially implies that $\Gamma$ is $<O R D$ stationary.

Definition 17. Let $\Gamma \subseteq \wp_{\kappa}(V)$. We say that $w C C(\Gamma)$ holds iff

(a) $\Gamma$ is $<$ ORD stationary; and

(b) There are stationarily many $\alpha<\kappa$ such that

$$
\sup \left\{\operatorname{otp}(X \cap O R D) \mid X \in \Gamma \wedge X \prec \Sigma_{1}(V, \in) \wedge X \cap \kappa=\alpha\right\} \geq \alpha^{+}
$$

The principle $w C C^{*}(\Gamma)$ is defined similarly, except (11) is replaced by:

$$
\left|\bigcup\left\{H_{X} \mid X \in \Gamma \wedge X \prec_{\Sigma_{1}}(V, \in) \wedge X \cap \kappa=\alpha\right\}\right| \geq \alpha^{+}
$$

Observation 18. If $\Gamma \subseteq \wp_{\kappa}(V)$, the principle $w C C(\Gamma)$ implies the principle $w C C^{*}(\Gamma)$, since

$$
\begin{array}{r}
\{\operatorname{otp}(X \cap O R D) \mid X \in \Gamma \text { and } X \cap \kappa=\alpha\} \\
=\left\{h e i g h t\left(H_{X \cap O R D}\right) \mid X \in \Gamma \text { and } X \cap \kappa=\alpha\right\}
\end{array}
$$

Definition 19. Suppose $\kappa$ is regular and uncountable and that $\Gamma \subseteq\{X \mid X \cap \kappa \in \kappa\}$. For each $\alpha<\kappa$ define:

$$
A_{\alpha}^{\Gamma}:=\bigcup\left\{H_{M} \mid M \in \Gamma \text { and } M \cap \kappa=\alpha\right\}
$$

Define the map $F^{\Gamma}$ with domain $\kappa$ by $\alpha \mapsto A_{\alpha}^{\Gamma}$.

Lemma 20. Suppose $\Gamma$ is a $<$ ORD-stationary (resp. club) subclass of $\wp_{\kappa}(V)$, and let $F^{\Gamma}$ be as in Definition 19. Then:

(1) $F^{\Gamma}$ satisfies Requirement $(A)$ in the Definition 8 of $a \diamond_{\text {Lav }}^{-}(\Gamma)$ function.

(2) There is some $\theta$ such that $F^{\Gamma}=F^{\Gamma \cap \wp_{\kappa}\left(H_{\theta}\right)}$

(3) Suppose $w C C^{*}(\Gamma)$ fails. Then $F^{\Gamma}$ also satisfies requirement $(B)$ of Definition 8, and thus $F^{\Gamma}$ is a $\diamond_{\text {Lav }}^{-}(\Gamma)$ (resp. $\diamond_{\text {Lav }}^{+}(\Gamma)$ ) function.

Proof. Consider any set $b$ and any structure $\mathfrak{A}=\left(H_{\theta}, \in,\{b\}, \ldots\right)$ in a countable language. Let $S:=\{M \in \Gamma \mid M \prec \mathfrak{A}\}$. Then for every $M \in S: 9$

$$
\sigma_{M}^{-1}(b) \in H_{M} \subseteq A_{M \cap \kappa}^{\Gamma}=F^{\Gamma}(M \cap \kappa)
$$

Part 2 just follows from the class Pigeonhole Principle and the definition of $A_{\alpha}^{\Gamma}$ in (13).

Finally, suppose ${ } \mathrm{CC}^{*}(\Gamma)$ fails. Then $\left|F^{\Gamma}(\alpha)\right|<\alpha^{+}$for almost every $\alpha<\kappa$. Combined with item (11) of the current lemma, this implies that $F^{\Gamma}$ is a $\diamond_{\text {Lav }}^{-}(\Gamma)$ function (in the case that $\Gamma$ was $<O R D$ stationary) or a $\diamond_{\text {Lav }}^{+}(\Gamma)$ function (in the case that $\Gamma$ was $<O R D$-club).

Corollary 21. Suppose $\kappa$ is regular uncountable and $\Gamma$ is a $<$ ORD stationary (resp. club) subclass of $\wp_{\kappa}(V)$ such that $w C C^{*}(\Gamma)$ fails. Then $\diamond_{\text {Lav }}(\Gamma)$ (resp. $\diamond_{\text {Lav }}^{+}(\Gamma)$ ) holds.

\footnotetext{
${ }^{8}$ Note $S$ is stationary or contains a club, depending on whether we assume $\Gamma$ is $<O R D$ stationary or $<O R D$ club.
} 
Proof. This follows immediately from Lemma 20 and Theorem 14.

The following remark shows that the converse of Corollary 21 is false (however, Question 34 asks whether a natural variation of the converse must hold).

Remark 22. The converse of Corollary 21 is false; i.e. it is possible for $\nabla_{\text {Lav }}(\Gamma)$ to and $w C C(\Gamma)$ to simultaneously hold. Suppose $\kappa$ is a supercompact cardinal, $\Gamma=$ $\wp_{\kappa}(V)$, and $F: \kappa \rightarrow V_{\kappa}$ is a (classical) supercompact Laver function. Then $w C C(\Gamma)$ holds.

It is also possible to obtain a counterexample to the converse of Corollary 27 where $\kappa$ is a successor cardinal. Suppose $\kappa$ is supercompact and $F: \kappa \rightarrow V_{\kappa}$ is a (classic) Laver function; suppose also that $\kappa$ is almost huge. Let $\mathbb{P}$ be any $\kappa$-cc forcing which is a subset of $V_{\kappa}$ and turns $\kappa$ into a successor cardinal, and let $G$ be $(V, \mathbb{P})$-generic. It is easy to see that the function $\tilde{F}: \kappa \rightarrow H_{\kappa}^{V[G]}$ defined by

$$
\alpha \mapsto(F(\alpha))_{G \cap V_{\alpha}}
$$

is a $\diamond_{\text {Lav }}(\kappa)$ function in $V[G] 9$ But the almost hugeness of $\kappa$ in $V$ implies that, in $V[G]$, the class $\wp_{\kappa}(V[G])$ has the wCC property.

Abstract Condensation principles have been extensively studied, for example by Law [14], Woodin [21], and Friedman-Holy [10]. Friedman and Holy considered several versions of Condensation, and proved that Stationary Condensation (even stronger versions called Local Club Condensation) are consistent with $\kappa$ being a very large cardinal. This is to be contrasted with the severe restraints that Club Condensation (as in [21]) place on the large cardinal properties of $\kappa$.

Definition 23. Suppose $\kappa$ is a regular uncountable cardinal. Stationary (resp. Club) Condensation holds at $\kappa$ iff there exists an $\in$-increasing and $\subseteq$-continuous sequence $\left\langle M_{\eta} \mid \eta<\kappa\right\rangle$ of transitive sets such that, letting

$$
\Gamma_{\vec{M}}:=\left\{X \in \wp_{\kappa}(V) \mid(\exists \eta<\kappa)\left(H_{X}=M_{\eta}\right)\right\}
$$

then for every regular $\theta \geq \kappa$, the set $\Gamma_{\vec{M}} \cap \wp_{\kappa}\left(H_{\theta}\right)$ is stationary (resp. Club).

They also proved:

Theorem 24. [Friedman-Holy [10]] Suppose $\kappa$ is regular. Then there is a $\kappa$-directed closed class forcing extension which satisfies ZFC and Stationary Condensation at $\kappa$ (and all larger cardinals).

The forcing first forces GCH above $\kappa$ and then performs a reverse Easton iteration of adding Cohen subsets of cardinal successors above $\kappa$.

Remark 25. All of the results of this section actually hold using a weaker, nonlinear form of condensation. In particular, for the Stationary Condensation theorems about a successor cardinal $\kappa$, all we need is some stationary $\Gamma \subseteq \wp_{\kappa}(V)$ such that for most $\alpha<\kappa$, the map

$$
s_{\alpha}^{\Gamma}:\{X \in \Gamma \mid X \cap \kappa=\alpha\} \rightarrow H_{\kappa}
$$

\footnotetext{
${ }^{9}$ In fact $\tilde{F}$ is a $\diamond_{\text {Lav }}(\Gamma)$ function for any class $\Gamma$ which has the property that for every $\theta$ there is a (possibly illfounded) generic embedding $i: V[G] \rightarrow W$ such that $i\left[H_{\theta}^{V[G]}\right] \in i(\Gamma)$.
} 
defined by

$$
X \mapsto H_{X}
$$

is at most $|\alpha|$-to-one.

Lemma 26. Suppose $\vec{M}=\left\langle M_{\eta} \mid \eta<\kappa\right\rangle$ is an $\in$-increasing, $\subseteq$-continuous sequence of (transitive) sets. Let $T \subseteq \Gamma_{\vec{M}}$, where $\Gamma_{\vec{M}}$ is defined as in (14). Then:

$$
w C C(T) \Longleftrightarrow w C C^{*}(T)
$$

Proof. The $\Leftarrow$ direction of (15) is obvious, by Observation 18. For the $\Rightarrow$ direction we show the contrapositive: assume $\mathrm{wCC}^{*}(T)$ holds, and consider a typical $\alpha<\kappa$ which witnesses this fact. Since $\vec{M}$ is $\in$-increasing then the map $\eta \mapsto \operatorname{height}\left(M_{\eta}\right)$ is one-to-one; it follows that for any pair $X, Y \in T$ :

$$
\operatorname{otp}(X \cap \theta)=\operatorname{otp}(Y \cap \theta) \Longrightarrow H_{X}=H_{Y}
$$

Thus since the set

$$
\left\{H_{X} \mid X \in T \text { and } X \cap \kappa=\alpha\right\}
$$

has cardinality $\geq \alpha^{+}$, then so does the set

$$
\left\{\operatorname{height}\left(H_{X}\right) \mid X \in T \text { and } X \cap \kappa=\alpha\right\}
$$

which means that $\alpha$ witnesses that $\mathrm{wCC}(T)$ holds.

Corollary 27. Assume Stationary Condensation (resp. Club Condensation) holds at $\kappa$, as witnessed by some $\vec{M}=\left\langle M_{\eta} \mid \eta<\kappa\right\rangle$. Let $\Gamma \subseteq \wp_{\kappa}(V)$ be the class of structures that condense to $\vec{M}$. Then:

$$
\neg w C C(\Gamma) \Longrightarrow \diamond_{\text {Lav }}(\Gamma)\left(\text { resp. } \diamond_{\text {Lav }}^{+}(\Gamma)\right)
$$

Proof. This follows directly from Corollary 21 and Lemma 26.

Lemma 28. If $\kappa$ is a successor cardinal and Stationary Condensation holds at $\kappa$ as witnessed by some $\vec{M}=\left\langle M_{\eta} \mid \eta<\kappa\right\rangle$, then $w C C^{*}\left(\Gamma_{\vec{M}}\right)$ fails.

Proof. Say $\kappa=\mu^{+}$. Let $\alpha \in\left(\mu, \mu^{+}\right)$; then there is some $\eta_{\alpha}<\kappa$ such that $M_{\eta_{\alpha}} \models$ $\alpha \notin \mathrm{CARD} 10$ If $X \prec_{\Sigma_{1}}(V, \in)$ and $\alpha=X \cap \kappa$ then $H_{X} \models \alpha \in \mathrm{CARD}$. It follows that

$$
R_{\alpha}:=\left\{H_{X} \mid X \in \Gamma_{\vec{M}} \text { and } X \prec_{\Sigma_{1}}(V, \in) \text { and } X \cap \kappa=\alpha\right\} \subset M_{\eta_{\alpha}}
$$

and so

$$
\left|R_{\alpha}\right| \leq\left|M_{\eta_{\alpha}}\right|<\kappa=\alpha^{+}
$$

Corollary 29. L satisfies $\diamond_{\text {Lav }}^{+}(\kappa)$ whenever $\kappa$ is a successor cardinal.

Proof. This follows from Lemma 28 and Corollary 27, note that $L$ satisfies Club Condensation.

\footnotetext{
${ }^{10}$ To see this: let $f: \mu \rightarrow \alpha$ be surjective. Let $X \prec\left(H_{\kappa}, \in,\{f\}\right)$ with $\alpha \subset X$; then $X=H_{X}=$ $M_{\eta}$ for some $\eta$, and sees that $\alpha$ is not a cardinal.
} 
5. Strengthening and simplification of main theorem from Viale [19]

The key ingredient of Viale's [19] proof of that PFA implies $\diamond_{\text {Lav }}\left(\omega_{2}\right)$ is his "Isomorphism Theorem" about a particular subclass of $\wp_{\omega_{2}}(V)$ which was shown by Viale-Weiß [20] to be stationary in $\wp_{\omega_{2}}\left(H_{\theta}\right)$ for all $\theta$ (under the assumption of PFA). This subclass of $\wp \omega_{2}(V)$ is called the class of $\omega_{1}$-internally club, $\omega_{1}$-guessing models, and denoted $\mathrm{GIC}_{\omega_{1}}$. The stationarity of this class is responsible for much of the consistency strength and many of the consequences of PFA, and is widely conjectured to be equiconsistent with PFA 11 We will not need to define GIC $_{\omega_{1}}$, but only use a few of its key properties.

For transitive $Z F^{-}$models $H$ and $H^{\prime}$, we say that $H$ is a hereditary initial segment of $H^{\prime}$ iff either $H=H^{\prime}$ or $H=\left(H_{\lambda}\right)^{H^{\prime}}$ for some $\lambda \in \mathrm{CARD}^{H^{\prime}}$.

Definition 30. $A$ class $\Gamma$ has the $\kappa$-Isomorphism Property iff whenever $X, X^{\prime}$ are elements of $\Gamma$ and $X \cap \kappa=X^{\prime} \cap \kappa$, then one of $H_{X}, H_{X^{\prime}}$ is a hereditary initial segment of the other.

The class GIC $_{\omega_{1}}$ easily projects downward (in the sense of Definition 13) 12 and Viale proved:13

Theorem 31. [Viale] Assume $H$ and $H^{\prime}$ are transitive $Z F^{-}$models such that $H \cap$ $H_{\omega_{1}}=H^{\prime} \cap H_{\omega_{1}}$, and $H, H^{\prime}$ are both in $G I C_{\omega_{1}}$. Then one of $H, H^{\prime}$ is a hereditary initial segment of the other.

Corollary 32. [Viale] Suppose $\mathfrak{c}=\omega_{2}$ and $p: \omega_{2} \leftrightarrow H_{\omega_{1}}$ is a bijection. Then

$$
\Gamma:=\bigcup_{\theta \geq \omega_{2}}\left\{X \in G I C_{\omega_{1}} \mid X \prec\left(H_{\theta}, \in, p\right)\right\}
$$

satisfies the $\omega_{2}$-Isomorphism Property.

Proof. This follows immediately from Theorem 31, since

$$
H_{X} \cap H_{\omega_{1}}=p\left[X \cap \omega_{2}\right]=p\left[X^{\prime} \cap \omega_{2}\right]=H_{X^{\prime}} \cap H_{\omega_{1}}
$$

Note also that the class $\Gamma$ from Corollary 32 is $\Pi_{1}(V)$ definable from the parameter $p \in H_{\omega_{3}}$. This parameter $p$ itself-i.e. this wellorder of $H_{\omega_{2}}$ in ordertype $\omega_{2}$-is not assumed to be definable in any way for our proof below of Theorem 1, Viale's original construction of a $\nabla_{\text {Lav }}\left(\omega_{2}\right)$ function made use of a definable wellorder of $H_{\omega_{2}}$ that exists under PFA, as proved by Caicedo-Velickovic [1]. We show that this coding mechanism turns out to be unnecessary, and we also provide a simplified, direct construction of the Laver function.

\footnotetext{
${ }^{11}$ Even without the "internally club" part.

${ }^{12}$ See Lemma 10 of [3].

${ }^{13} \mathrm{~A}$ simplified and elementary proof of the Isomorphism Theorem for $G I C_{\omega_{1}}$ can be found in Section 2.3 of Cox-Viale [3].
} 
Remark 33. The conjunction of $\mathfrak{c}=\omega_{2}$ and stationarity of $G I C_{\omega_{1}}$ is strictly weaker than PFA. It follows from PFA by Todorcevic [18] and Viale-Weiß [20]; but it does not imply PFA as shown by two different constructions 14

(1) the author has shown that the model obtained by forcing with Neeman's [15] pure side condition poset using models of 2 types below a supercompact cardinal produces a model of $\mathfrak{c}=\omega_{2}$ plus stationarity of $G I C_{\omega_{1}}$ which is not a model of PFA.

(2) Menachem Magidor has shown that adding a Cohen real over an arbitrary model of PFA preserves the stationarity of $G_{1} C_{\omega_{1}}$; and by Shelah [16] this forcing extension does not even model Martin's Axiom.

5.1. Proof of Theorem 1, Suppose $\Gamma$ is a stationary subclass of $\wp_{\kappa}(V)$ satisfying the assumptions of Theorem 1, i.e. it projects downward, it satisfies the $\kappa$-Isomorphism Property, and it is $\Pi_{1}(V)$ definable from some parameter $p$, where $p \in H_{\kappa^{+}}$. Given a function $F: \kappa \rightarrow H_{\kappa}$, let us say that a set $b$ is a witness to non- $\Gamma$-Laverness of $F$ iff there is some algebra $\mathcal{A}_{b}=\left(H_{|\operatorname{trcl}(b)|^{+}}, \in,\{p, b\}, \ldots\right)$ such that $\sigma_{M}^{-1}(b) \neq F(M \cap \kappa)$ for every $M \in \Gamma \cap \wp_{\kappa}\left(H_{\theta}\right)$ such that $M \prec \mathcal{A}_{b}$. We say that a regular cardinal $\theta$ is the least cardinal witnessing non- $\Gamma$-Laverness of $F$ iff $\theta$ is the least regular cardinal such that there is a $b \in H_{\theta}$ witnessing the non- $\Gamma$-Laverness of $F$.

For any $\alpha<\kappa$ and any partial $g: \alpha \rightarrow H_{\kappa}$, let $\mathcal{W}_{g}^{\Gamma}$ be the set of transitive $Z F^{-}$ models $W$ such that:

- there is an elementary $\sigma: W \rightarrow_{\Sigma_{1}} V$ with $\alpha=\operatorname{crit}(\sigma)$ and $\sigma(\alpha)=\kappa$;

- $\operatorname{range}(\sigma) \in \Gamma$ and $p \in \operatorname{range}(\sigma)$;

- $W$ has a largest cardinal $\theta_{W}$

- $g \in W$ and $W$ believes that $\theta_{W}$ is the least regular cardinal witnessing non$\Gamma^{W}$-Laverness of $g$, where $\Gamma^{W}$ is the subset of $W$ defined over $W$ using the definition of $\Gamma$ and the parameter $\sigma^{-1}(p)$.

Claim 33.1. For any $\alpha<\kappa$ and any $g: \alpha \rightarrow H_{\kappa}$, the set $\mathcal{W}_{g}^{\Gamma}$ has at most one element.

Proof of Claim 33.1. Suppose $W, W^{\prime}$ were two distinct elements of $\mathcal{W}_{g}^{\Gamma}$; let $\sigma$ : $W \rightarrow_{\Sigma_{1}} V$ and $\sigma^{\prime}: W^{\prime} \rightarrow_{\Sigma_{1}} V$ be the maps required by the definition of $\mathcal{W}_{g}^{\Gamma}$, and set $M:=\operatorname{range}(\sigma)$ and $M^{\prime}:=\operatorname{range}\left(\sigma^{\prime}\right)$. Note that $p \in M \cap M^{\prime} \cap H_{\kappa^{+}}$, and it follows easily (by coding $p$ as a subset of $\kappa$ in an absolute manner and using the assumption that $\left.M \cap \kappa=M^{\prime} \cap \kappa=\alpha\right)$ that $p_{W}:=\sigma^{-1}(p)$ is equal to $p_{W^{\prime}}:=\sigma^{\prime}-1(p)$. By the assumption that $\Gamma$ has the $\kappa$-Isomorphism Property, one of $W, W^{\prime}$ is a hereditary initial segment of the other; WLOG assume $W$ is a strict hereditary initial segment of $W^{\prime}$. Note then that $\theta_{W}$ (the largest cardinal of $W$ ) is strictly smaller than $\theta_{W^{\prime}}$ (the largest cardinal of $W^{\prime}$ ). Note also, since $p_{W}=p_{W^{\prime}}, \Gamma$ is $\Pi_{1}$ definable in $p$, and $W$ is a hereditary initial segment of (in particular a $\Sigma_{1}$ elementary substructure of) $W^{\prime}$, then

$$
\Gamma^{W}=\Gamma^{W^{\prime}} \cap W
$$

\footnotetext{
${ }^{14}$ Each construction also shows that the conjunction of $\mathfrak{c}=\omega_{2}$ with stationarity of $G I C_{\omega_{1}}$ does not imply the existence of the Caicedo-Velickovic coding.
} 
Let $\bar{H}:=\left(H_{\theta_{W}}\right)^{W}=\left(H_{\theta_{W}}\right)^{W^{\prime}}$. Then

$$
X:=\left(\wp_{\alpha}(\bar{H})\right)^{W} \cap \Gamma^{W}
$$

Let $b \in \bar{H}$ witness (from the point of view of $W$ ) that $g$ is not a $\diamond_{\mathrm{Lav}}\left(\Gamma^{W}\right)$ function. Then there is some algebra $\mathcal{A}_{b} \in W$ on $\bar{H}$ such that:

$$
W \models(\forall M \in X)\left(M \prec \mathcal{A}_{b} \Longrightarrow \sigma_{M}^{-1}(b) \neq g(M \cap \alpha)\right)
$$

This is a $\Sigma_{1}$ statement in the parameters $X, \mathcal{A}_{b}$, and $g$, and is thus upward absolute to $W^{\prime}$; but since $X$ is also equal to $\left(\wp_{\alpha}(\bar{H})\right)^{W^{\prime}} \cap \Gamma^{W^{\prime}}$, this contradicts the minimality of $\theta_{W^{\prime}}$.

Now for any $\alpha<\kappa$ and any $g: \alpha \rightarrow H_{\kappa}$, let $W_{g}$ denote the unique element of $W_{g}^{\Gamma}$ given by Claim 33.1 (if it exists) and let $\theta_{g}$ the largest cardinal of $W_{g}$. Let $b_{g}$ be any witness in $\left(H_{\theta_{g}}\right)^{W_{g}}$ to the non-Laverness of $g$ w.r.t. $\Gamma^{W_{g}}$.

Claim 33.2. The function $F: \kappa \rightarrow H_{\kappa}$ defined recursively by $\alpha \mapsto b_{g \mid \alpha}$ (if this exists; 0 otherwise) is a $\diamond_{\text {Lav }}(\Gamma)$ function.

Proof of Claim 33.2. This is where we use assumption that $\Gamma$ projects downward, along with the assumption that $\Gamma$ is stationary at every $\wp_{\kappa}\left(H_{\Omega}\right)$. Suppose for a contradiction that $F$ is not a $\diamond_{\text {Lav }}(\Gamma)$ function; let $\theta$ be the least regular cardinal witnessing non- $\Gamma$-Laverness of $F$. Let $\Omega:=\theta^{+}$and, using stationarity of $\Gamma$, pick an $M^{\prime} \prec\left(H_{\Omega}, \in,\{F, p\}\right)$ such that $M^{\prime} \in \Gamma$. Let $\alpha:=M^{\prime} \cap \kappa, \sigma_{M^{\prime}}: H_{M^{\prime}} \rightarrow M^{\prime}$ be the inverse of the Mostowski collapse of $M^{\prime}$, and $\bar{\Gamma}:=\sigma_{M^{\prime}}^{-1}\left(\Gamma \cap \wp_{\kappa}\left(H_{\theta}\right)\right)$. Then $\sigma_{M^{\prime}}^{-1}(F)=F\left\lceil\alpha\right.$ and $H_{M^{\prime}}$ is the unique element of $\mathcal{W}_{F\lceil\alpha}^{\Gamma}$; so by the recursive definition of the function $F$, we know that $F(\alpha)$ is some element of $\bar{H}$ witnessing the non- $\bar{\Gamma}$-Laverness of $F\left\lceil\alpha\right.$ from the point of view of $H_{M^{\prime}}$. Set $\bar{b}:=F(\alpha) \in H_{M^{\prime}}$ and let $\overline{\mathcal{A}}=(\bar{H}, \in,\{\bar{b}, \bar{p}\}, \ldots) \in H_{M^{\prime}}$ be an algebra corresponding to the witness $\bar{b}$. Let $b:=\sigma_{M^{\prime}}(\bar{b})$ and $\mathcal{A}:=\sigma_{M^{\prime}}(\overline{\mathcal{A}})$; by elementarity of $\sigma_{M^{\prime}}$ :

$$
H_{\Omega} \models(\forall M)\left(M \prec \mathcal{A} \wedge M \in \Gamma \Longrightarrow \sigma_{M}^{-1}(b) \neq F(M \cap \kappa)\right)
$$

Set $M:=M^{\prime} \cap H_{\theta}$; by the downward projection assumption on the class $\Gamma$, we know that

$$
M \in \Gamma
$$

Furthermore, since $M^{\prime} \prec\left(H_{\Omega}, \in,\{\mathcal{A}\}\right)$ then

$$
M=M^{\prime} \cap H_{\theta} \prec \mathcal{A}
$$

Finally:

$$
\sigma_{M}^{-1}(b)=\sigma_{M^{\prime}}^{-1}(b)=\bar{b}=F(\alpha)=F\left(M^{\prime} \cap \kappa\right)=F(M \cap \kappa)
$$

These properties of $M$ contradict (16), and complete the proof of the claim.

This completes the proof of Theorem 1. 


\section{Concluding Remarks}

Recall that Corollary 27 said that if $\Gamma \subseteq \wp_{\kappa}(V)$ and the appropriate version of Chang's Conjecture fails for $\Gamma$, then $\diamond_{\text {Lav }}(\Gamma)$ holds; and Remark 22 demonstrated that the converse was not literally true. However, it's still natural to wonder if $\diamond_{\text {Lav }}(\Gamma)$ must always be essentially due to some failure of Chang's Conjecture:

Question 34. Suppose $\kappa$ is a successor cardinal, $\Gamma \subset \wp_{\kappa}(V)$, and $F: \kappa \rightarrow H_{\kappa}$ is a $\diamond_{\text {Lav }}(\Gamma)$ function. Must there be some (definable) $\Gamma^{\prime} \subseteq \Gamma$ such that:

(1) $F$ is still $a \diamond_{\text {Lav }}\left(\Gamma^{\prime}\right)$ function; and

(2) $w C C\left(\Gamma^{\prime}\right)$ fails?

\section{REFERENCES}

[1] Andrés Eduardo Caicedo and Boban Veličković, The bounded proper forcing axiom and well orderings of the reals, Math. Res. Lett. 13 (2006), no. 2-3, 393-408. MR2231126 (2007d:03076)

[2] Paul Corazza, Laver sequences for extendible and super-almost-huge cardinals, J. Symbolic Logic 64 (1999), no. 3, 963-983, DOI 10.2307/2586614. MR1779746 (2001g:03096)

[3] Sean Cox and Matteo Viale, Martin's Maximum and tower forcing, Israel J. Math. 197 (2013), no. 1, 347-376, DOI 10.1007/s11856-013-0004-0. MR3096619

[4] Hans-Dieter Donder and Jean-Pierre Levinski, Some principles related to Chang's conjecture, Ann. Pure Appl. Logic 45 (1989), no. 1, 39-101, DOI 10.1016/0168-0072(89)90030-4. MR1024901 (91b:03087)

[5] Hans-Dieter Donder and Pierre Matet, Two cardinal versions of diamond, Israel J. Math. 83 (1993), no. 1-2, 1-43, DOI 10.1007/BF02764635. MR1239715 (94k:03065)

[6] Joel David Hamkins, A class of strong diamond principles, Preprint (2002), available at math/0211419.

[7] Alex Hellsten, Diamonds on large cardinals, Ann. Acad. Sci. Fenn. Math. Diss. 134 (2003), 48. Dissertation, University of Helsinki, Helsinki, 2003. MR2026390 (2004j:03054)

[8] Thomas J. Jech, Some combinatorial problems concerning uncountable cardinals, Ann. Math. Logic 5 (1972/73), 165-198. MR0325397 (48 \#3744)

[9] Matthew Foreman and Menachem Magidor, Large cardinals and definable counterexamples to the continuum hypothesis, Ann. Pure Appl. Logic 76 (1995), no. 1, 47-97. MR1359154 (96k:03124)

[10] Sy-David Friedman and Peter Holy, Condensation and large cardinals, Fund. Math. 215 (2011), no. 2, 133-166, DOI 10.4064/fm215-2-3. MR2860182

[11] Moti Gitik and Saharon Shelah, On certain indestructibility of strong cardinals and a question of Hajnal, Arch. Math. Logic 28 (1989), no. 1, 35-42, DOI 10.1007/BF01624081. MR987765 (90e:03063)

[12] Kenneth Kunen, Set theory, Studies in Logic and the Foundations of Mathematics, vol. 102, North-Holland Publishing Co., Amsterdam, 1980. An introduction to independence proofs. MR597342 (82f:03001)

[13] Richard Laver, Making the supercompactness of $\kappa$ indestructible under $\kappa$-directed closed forcing, Israel J. Math. 29 (1978), no. 4, 385-388. MR0472529 (57 \#12226)

[14] David Richard Law, An abstract condensation property, ProQuest LLC, Ann Arbor, MI, 1994. Thesis (Ph.D.)-California Institute of Technology. MR2691107

[15] Itay Neeman, Forcing with sequences of models of two types (preprint).

[16] Saharon Shelah, Can you take Solovay's inaccessible away?, Israel J. Math. 48 (1984), no. 1, 1-47, DOI 10.1007/BF02760522. MR768264 (86g:03082a)

[17] Masahiro Shioya, Splitting $\mathcal{P}_{\kappa} \lambda$ into maximally many stationary sets, Israel J. Math. 114 (1999), 347-357, DOI 10.1007/BF02785587. MR1738689 (2001i:03100)

[18] Stevo Todorčević, Partition problems in topology, Contemporary Mathematics, vol. 84, American Mathematical Society, Providence, RI, 1989. MR980949 (90d:04001) 
[19] Matteo Viale, Guessing models and generalized Laver diamond, Ann. Pure Appl. Logic 163 (2012), no. 11, 1660-1678, DOI 10.1016/j.apal.2011.12.015.

[20] Matteo Viale and Christoph Weiß, On the consistency strength of the proper forcing axiom, Adv. Math. 228 (2011), no. 5, 2672-2687, DOI 10.1016/j.aim.2011.07.016. MR2838054 (2012m:03131)

[21] W. Hugh Woodin, The axiom of determinacy, forcing axioms, and the nonstationary ideal, de Gruyter Series in Logic and its Applications, vol. 1, Walter de Gruyter \& Co., Berlin, 1999. MR1713438 (2001e:03001)

E-mail address: scox9@vcu.edu

Department of Mathematics and Applied Mathematics, Virginia Commonwealth UniVersity, 1015 Floyd Avenue, Richmond, Virginia 23284, USA 